The radial force of the Pipeline was able to push a herniated WEB device into the aneurysm in all but one case where a balloon was employed to angioplasty the stent.

Conclusions We believe this is the first series reporting combined use of WEB and Pipeline for the treatment of intracranial aneurysms. Treatment failure rates of PED as a stand-alone treatment have been reported to be from $11.9 \%$ to $20 \%$. Intrasaccular flow diversion with the WEB device allows for increased metal coverage at both the aneurysm neck, preventing inflow, and the aneurysm dome, protecting from rupture. For larger aneurysms the WEB device theoretically protects from a destabilizing mural thrombus. Also, in larger aneurysm were WEB devices have been shown to be less enduring, endoluminal remodeling provides a durable treatment. This series demonstrates the WEB device as a feasible and safe alternative to adjuvant coiling for achieving immediate intrasaccular flow diversion when using the PED. Disclosures T. White: None. K. Shah: None. J. Turpin: None. J. Katz: None. H. Woo: None.

\section{E-237 RUPTURED BLISTER-TYPE CEREBRAL ANEURYSM TREATED WITH FLOW DIVERSION USING A NOVEL ANTIPLATELET AGENT CANGRELOR}

T Wilson*, D Ramanathan, J Dye. Neurosurgery, Loma Linda University, Loma Linda, CA

\subsection{6/neurintsurg-2020-SNIS.268}

Introduction Blister type aneurysms are challenging to treat surgically and endovascularly with the ideal treatment approach debated. Recently, endovascular procedures, specifically, flow diversion, gained popularity in treating blister aneurysms; however, the need for dual antiplatelet therapy arouses concern, especially in blister aneurysms with their high risk of intraprocedural rupture that may require further intervention. Cangrelor is a novel intravenous P2Y12 platelet receptor antagonist with reversible binding and rapid onset and offset of action. These characteristics are appealing for use in endovascular procedures and may mitigate some concerns associated with other P2Y12 receptor antagonists.

Case Summary This is a 42-year old female who presented with sudden onset of severe headache, nausea, and vomiting. On exam, she was drowsy with no focal neurologic deficits. Initial CT head, CT angiogram, and digital subtraction angiography (DSA) were negative for aneurysm or other vascular pathology. On post bleed day (PBD) 7, repeat DSA demonstrated severe vasospasm and a nonspecific ectatic segment along the distal intracranial internal carotid artery (ICA). On daily angiograms for intraarterial spasmolysis, the ectatic segment remained unchanged until PBD 14, when it began to enlarge and exhibited rapid growth over several days.

Management We treated this patient endovascularly using a pipeline flow diversion device. Immediately prior to pipeline placement, in addition to aspirin and heparin, we also began infusing cangrelor, a novel, reversible, intravenous P2Y12 platelet receptor antagonist with rapid onset and offset of action. After the procedure, she transitioned from cangrelor to prasugrel. Our patient tolerated the procedure well, and on postprocedure day 4, she was discharged home on daily prasugrel and aspirin. To date, our patient is doing well clinically, and her pipeline appears patent with no evidence of aneurysm recurrence.
Conclusion This is one of the first reports of the use of cangrelor in neuro-endovascular procedures. This is important as cangrelor has the potential for improving the safety of endovascular procedures especially in patients where treatment is associated with high risks of intraprocedural bleeding or other complications that may require further intervention, such as in treatment of blister aneurysms.

Disclosures T. Wilson: None. D. Ramanathan: None. J. Dye: None.

\section{E-238 RELATIVE ANGLE OF DEFLECTION CORRELATES WITH ANEURYSMAL RUPTURE STATUS IN POSTERIOR COMMUNICATING ARTERY ANEURYSMS}

${ }^{1} \mathrm{~N}$ Damodara*, ${ }^{2} \mathrm{~K}$ Thomas, ${ }^{3} \mathrm{~S}$ Quinn, ${ }^{1} \mathrm{C}$ Gandhi, ${ }^{4} \mathrm{C}$ Prestigiacomo. ${ }^{1}$ Neurosurgery, Westchester Medical Center, Valhalla, NY; ${ }^{2}$ Neurosurgery, Rutgers New Jersey Medical School, Newark, Nj; ${ }^{3}$ Neurosurgery, University of Texas Health Science Center, Houston, TX; ${ }^{4}$ Neurosurgery, University of Cincinnati College of Medicine, Cincinnati, $\mathrm{OH}$

\subsection{6/neurintsurg-2020-SNIS.269}

Background The ability to prognosticate rupture for cerebral aneurysms is paramount to prevent the risks inherent to open clipping or endovascular coiling. The goal of this study was to create a mathematical model to predict the probability of rupture incorporating the salient biomorphometric characteristics of the aneurysm.

Methods Posterior communicating artery aneurysms confirmed by computed tomography angiography were subjected to three-dimensional reconstruction to ascertain the following biomorphometric parameters: height, width, neck size, aspect ratio, bottle neck factor, aneurysm angle, deflection angle, neck angle, and proximal internal carotid artery- distal internal carotid artery angle. Significant factors related to rupture were determined and a forward stepwise binary logistic regression was performed to establish the log-odds of rupture.

Results A total of 101 aneurysms (80 ruptured and 21 unruptured) were included. Of the six statistically significant biomorphometric parameters measured, aneurysm deflection angle and aspect ratio both were considerably larger $(p=0.001)$ in ruptured cases compared to unruptured ones. Binary logistic regression applied to the dataset demonstrated a $96 \%$ sensitivity and $89 \%$ overall accuracy.

Conclusions This updated binary logistic regression model was able to identify aneurysm rupture more robustly when compared to previous models. Future studies combining patient specific characteristics, along with previously determined biomorphometric parameters may further enhance this model.

Disclosures N. Damodara: None. K. Thomas: None. J. Quinn: None. C. Gandhi: None. C. Prestigiacomo: None.

\section{E-239 INTRA-ANEURYSMAL FLOW AFTER TREATMENT WITH THE WOVEN ENDOBRIDGE (WEB) MEASURED BY QUANTITATIVE DIGITAL SUBTRACTION ANGIOGRAPHY}

K Shah*, T White, J Turpin, I Teron, J Katz, H Woo. Neurosurgery, Northwell Health, Manhasset, NY

\subsection{6/neurintsurg-2020-SNIS.270}

Introduction The Woven EndoBridge (WEB) is an intra-saccular flow-diverting device that is rapidly becoming popular in the treatment of wide-neck bifurcation aneurysms. However, 
several challenges remain, such as device compression, incomplete occlusion, and thromboembolic complications. Changes in intra-aneurysmal flow after the use of the WEB are not well understood and may be important in the performance of the device for aneurysm occlusion. The aim of this study was to characterize the degree of immediate aneurysm occlusion using color-coded quantitative digital subtraction angiography (DSA) after treatment with the WEB.

Materials and Methods We retrospectively reviewed records of 34 patients treated with the WEB between March 2019 and February 2020. Eight patients were excluded from analysis for the use of a concurrent stent or intra-luminal flow-diverter. Measurements of contrast transit times on DSA were calculated with syngo iFlow (Siemens Healthineers AG). Regions of interest were selected within the parent vessel immediately proximal to the aneurysm and within the aneurysm dome before and after treatment with WEB (figure 1). The ratio of aneurysm contrast transit time to parent vessel contrast transit time was obtained before WEB placement and immediately after WEB placement. Transit time ratios were also compared with pre- and post-Pipeline (PED) cases matched for aneurysm size.

Results Out of 26 aneurysms, 24 aneurysms demonstrated an increase in contrast transit time after WEB placement $(2.06 \mathrm{~s}$, $\mathrm{p}<0.05$ ), as measured by iFlow analysis. The ratio of aneurysm-to-parent vessel contrast transit time increased significantly after treatment with WEB $(1.02$ vs. $1.61 ; \mathrm{p}<0.01)$. Similarly, the ratio of contrast transit time increased after PED deployment $(1.07$ vs. $1.51 ; \mathrm{p}<0.01)$, however the ratio increased significantly more after WEB when compared to PED (67\% vs $40 \% ; \mathrm{p}<0.05)$. Average aneurysm size was similar between WEB cases $(n=26)$ and PED cases $(n=26) \quad\left(128.2 \mathrm{~mm}^{3}\right.$ vs $\left.165.1 \mathrm{~mm}^{3}, \mathrm{p}=0.52\right)$. The average number of PED devices used per case was 2.2.

Conclusions High rates of immediate aneurysm contrast stagnation can be achieved with use of the WEB. As a flowdiverting device, the WEB offers a greater degree of immediate aneurysm occlusion than the PED. iFlow analysis provides a quantitative measure of post-treatment effect and could represent a predictive tool for successful long-term occlusion.

Disclosures K. Shah: None. T. White: None. J. Turpin: None. I. Teron: None. J. Katz: None. H. Woo: None.

\section{E-240 SAFETY AND EFFICACY OF TRACSTAR LARGE DISTAL PLATFORM DURING ENDOVASCULAR TREATMENT OF INTRACRANIAL ANEURYSMS}

D Bageac*, R De Leacy. Neurosurgery, The Mount Sinai Hospital, New York, NY

10.1136/neurintsurg-2020-SNIS.271

Introduction Increased procedure time during endovascular treatment of cerebral aneurysms is associated with increased anesthesia related complications and radiation exposure. Elimination of the need for intermediate catheters during aneurysm treatment may decrease procedure time and increase cost effectiveness. A novel device -the TracStar Large Distal Platform (LDP) -may offer more distal final positioning when compared to commonly used guide catheters, thus decreasing the need for guide catheter use. We investigate the safety and efficacy of the TracStar LDP when used during endovascular aneurysm treatment.
Methods We perform a multicenter retrospective review of endovascular cerebral aneurysm embolizations during which the TracStar LDP was utilized. Aneurysm location, procedural information, and complications were recorded as detailed in the operative note. Vascular tortuosity was assessed via pre-procedural CTA. Distal-most position achieved with TracStar was determined by review of intraprocedural imaging.

Results A preliminary analysis of 30 cases was performed. Flow diversion was performed in 25 cases (83\%), and stent assisted coiling was performed in the remainder. The target aneurysm was located in the ICA in 27 cases (90\%), the MCA bifurcation in 2 cases (7\%), and the ACA in 1 case $(3 \%)$. An intermediate catheter was required in 18 cases (60\%). TracStar LDP achieved stable access to the Cavernous ICA in $70 \%$ of cases. Average procedure time was 67 \pm 33 minutes. There were no procedural complications or new neurologic defects after treatment. TracStar LDP was exchanged for an alternative guide catheter in one case due to catheter kinking, and in two cases due to lack of support.

Conclusion Use of Tracstar LDP during flow-diversion and sent-assisted coiling of cerebral aneurysms is safe and effective. Access to the cavernous segment of the ICA was achieved in the majority of cases, and use of an intermediate catheter was not required in $40 \%$ of cases. Final analysis of the the full 60 patient multi-center study will be available for presentation at SNIS Annual 2020.

Disclosures D. Bageac: None. R. De Leacy: None.

\section{E-241 A PROSPECTIVE, MULTICENTER STUDY ASSESSING THE EMBOLIZATION OF INTRACRANIAL ANEURYSMS USING WAVE'M EXTRA SOFT COILS, A PART OF THE PENUMBRA SMART COIL ${ }^{\circledR}$ SYSTEM: STUDY PROTOCOL FOR SURF}

${ }^{1} \mathrm{C}$ Schirmer*, ${ }^{2}$ I Kaminsky, ${ }^{3} \mathrm{M}$ Chaudry, ${ }^{4} \mathrm{O}$ Zaidat, ${ }^{5} \mathrm{~A}$ Alshekhlee, ${ }^{6} \mathrm{P}$ Ramakrishnan, ${ }^{7} \mathrm{M}$ Taqi. ${ }^{1}$ Geisinger, Wilkes-Barre, PA; ${ }^{2}$ Radiology Imaging Associates, Englewood, CO; ${ }^{3}$ Prisma Health, Greenville, SC; ${ }^{4}$ Mercy Health - St. Vincent, Toledo, $\mathrm{OH}_{;}{ }^{5} \mathrm{SSM}$ Health, Bridgeton, MO; ${ }^{6}$ Riverside Regional Medical Center, Newport News, VA; ${ }^{7}$ Los Robles, Thousand Oaks, CA

\subsection{6/neurintsurg-2020-SNIS.272}

Introduction/Purpose Initial clinical evidence has shown that coiling with the SMART COIL ${ }^{\circledR}$ System (Penumbra, Inc.) is a safe and durable treatment option for intracranial aneurysms. ${ }^{1-}$ 5 The WAVE ${ }^{\mathrm{TM}}$ Extra Soft Coil (WAVE) is part of the SMART Coil System and is specifically designed as a fill and finish coil. The primary objective of the SURF study, a post-market registry, is to assess the utility of WAVE as a fill and finish coil to support adequate occlusion at one year follow-up. A secondary objective is to compare the ability of digital subtraction angiography (DSA) and magnetic resonance angiography (MRA) to detect incomplete occlusion in the coiled aneurysms.

Materials and Methods SURF is a post-market, prospective, multicenter, single-arm, observational study that will enroll approximately 800 consecutive patients at up to 50 centers in North America. Patients age $\geq 18$ years, having embolization of intracranial aneurysms, with WAVE as the final finishing coil and Penumbra SMART COIL System accounting for at least $75 \%$ of total number of coils implanted, will be 